\title{
Autoeficacia de profesores de educación física para la inclusión de alumnos con discapacidad y su relación con la formación y el contacto previo
}

\author{
Raúl REINA ${ }^{1}$, Ilse HEMMELMAYR y Beatriz SIERRA-MARROQUÍN \\ Universidad Miguel Hernández de Elche, España
}

(Recibido 27 Mayo, 2016; Aceptado 16 Junio, 2016)

\begin{abstract}
RESUMEN: La formación y experiencias positivas previas son fundamentales para una adecuada percepción de competencia, confianza y actitud positiva para la inclusión de alumnos con discapacidad en educación física. Este trabajo analiza la fiabilidad de una escala aplicada al contexto español para evaluar las creencias de autoeficacia para atender a alumnos con discapacidad intelectual, física y visual, mostrando excelentes valores de fiabilidad y consistencia interna la Escala de Autoeficacia en Profesores de Educación Física hacia Alumnos con Discapacidad (EA-PEF-AD). Los 102 profesores que participaron en el estudio mostraron además niveles de autoeficacia más altos $(\mathrm{p}<0.001)$ a medida que habían tenido formación y experiencias profesionales previas. Los resultados del presente trabajo confirmarían la idoneidad de llevar a cabo programas formativos para profesores de educación física en activo, preferiblemente con contacto con personas con discapacidad.
\end{abstract}

Palabras clave: enseñanza, competencia, actitud.

\section{Self-efficacy of Physical Education teachers toward inclusion of students with disabilities and regarding their previous training and experiences}

\begin{abstract}
Previous training and past positive experiences are key factors to develop an adequate self-competence, self-confidence and positive attitudes toward inclusion of students with disabilities into physical education. This study analyses the reliability of a Spanish version of the Self-Efficacy Scale for Physical Education Teacher Education Majors towards Children with Disabilities (SEPETE-D). One-hundred and two physical education professors participated in this study. Results indicated that professors scored higher on self-efficacy $(\mathrm{p}<0.001)$ if they received previous training in adapted physical education or they had previous professional experiences on this regard. Our results recommend providing training opportunities for physical educators who are currently working in schools, with the involvement of persons with disabilities.
\end{abstract}

Keywords: education, self-competence, attitude.

${ }^{1}$ Correspondencia: Universidad Miguel Hernández de Elche, Centro de Investigación del Deporte. Avda. de la Universidad s/n, 03202, Elche. E-mail: rreina@goumh.es. 
España ratificó la Convención de las Naciones Unidas (ONU, 2006) acerca de los derechos de las Personas con Discapacidad en 2007, cuyo artículo 24 establece que la inclusión de alumnos con discapacidad en el sistema educativo debería ser la norma y no una excepción (United Nations, 2015). En el curso 2015-2016, la Ley Orgánica para la Mejora de la Calidad Educativa (LOMCE, 2013) comenzó a implantarse en España, postulando que la adquisición de competencias y conocimientos debe basarse en un modelo educativo que estimule la autonomía de los estudiantes, con un énfasis en metodologías cooperativas y sociales, que incluya la promoción de valores. Sin embargo, muchos de los programas formativos para profesores de educación física en España carecen de una formación específica en educación física inclusiva EFI- (Reina, Hutzler, Iniguez-Santiago, y Moreno-Murcia, 2016), con una especial preocupación en aquellos profesores que están en el sistema educativo y no tienen oportunidades para poder actualizar sus conocimientos o competencias en materia de inclusión.

La inclusión en entornos deportivos o que impliquen el movimiento, tales como la educación física, conlleva numerosos beneficios, con una mejor aceptación de las personas con diferentes capacidades en actividades de la vida diaria, mejora de la calidad de vida y aumento de su competencia social (Block, 1994; Reina, 2014). La inclusión permite interacciones entre alumnos de edades similares, reduciendo los sentimientos de aislamiento, incrementando las expectativas de las personas, cambiando actitudes o ayudando a la aceptación de diferencias, incrementando así los derechos de las personas con discapacidad y la igualdad de oportunidades (DePauw, y Doll-Tepper, 2000; Ocete-Calvo, 2016).

A pesar de todos los beneficios que la EFI reporta, encontramos en ocasiones que la implementación de la misma no es efectiva, cuando los profesores y profesoras de educación física son los responsables de realizar las adaptaciones necesarias para una participación exitosa, segura y, sobre todo, inclusiva. Así, varios trabajos de la literatura evidencian que una inadecuada formación o desarrollo profesional de los profesores es una de las barreras para una inclusión efectiva, unido a otros aspectos como el limitado apoyo a los profesores, las clases sobredimensionadas en cuanto al número de alumnos, el tiempo disponible para organizar y realizar la actividad educativa, la falta de competencia y/o la confianza percibida por parte de los profesores (Kodish, Kulinna, Martin, Pangrazi, y Darst, 2006; Konza, 2008; Block, y Obrushnikova, 2007; Block, Hutzler, Barak, y Klavina, 2013), aspecto éste último sobre el que centraremos el presente trabajo.

Una inadecuada preparación de los profesores conlleva un efecto negativo en la percepción de competencia, confianza y actitudes para con el proceso inclusivo (Ammah, y Hodge, 2005; Hutzler, Zach, y Gafni, 2005; Ocete-Calvo, 2016), por lo que la formación orientada para la mejora del sentimiento de autoeficacia se convierte en un aspecto fundamental. La teoría de la autoeficacia de Bandura (Bandura, 1989) es un elemento relevante de la Teoría Social-Cognitiva, sirviendo de marco de referencia para poder evaluar la autoconfianza percibida, en este caso de los profesores de EF para con la inclusión de alumnos con discapacidad en sus clases. De una forma más concreta, la autoeficacia es una forma de evaluación de la percepción de confianza en situaciones específicas, con el fin de poder dar una respuesta efectiva a las mismas. De acuerdo con Bandura (1989), las personas precisan creer tener el control sobre todo aquello que afecte a sus vidas, con el fin de conseguir los objetivos personales o profesionales que se proponen. 
Son pocos los trabajos en la literatura que han aplicado la teoría de la Autoeficacia en EFI (Ammah, y Hodge, 2005; Hutzler et al., 2005; Ocete-Calvo, 2016), la cual puede utilizarse para evaluar las creencias mostradas por los profesores durante su práctica profesional y predecir sus estrategias en la práctica (Kozub, y Lienert, 2003), siendo además un constructo válido para evaluar las creencias de auto-competencia del profesorado como un factor predictor de su actitud hacia la inclusión de alumnos con discapacidad (Taliaferro, Hammond, y Wyant, 2015).

Los trabajos de Hutzler et al. (2005) y de Jovanovic, Kudlacek, Block y Djordjevic(2014) mostraron la estrecha relación entre la autoeficacia y las actitudes positivas para incluir a estudiantes con discapacidad en educación física, encontrando además que las profesoras evidenciaron mayores niveles de autoeficacia que los profesores. Además, tales trabajos también mostraron que la previa participación en actividades relacionadas con las actividades físicas o deportes adaptados para personas con discapacidad se relacionaba con unos mayores niveles de autoeficacia.

Los objetivos de la presente investigación son: a) analizar la fiabilidad de una escala aplicada al contexto español para evaluar las creencias de autoeficacia mostrada por profesores y profesoras de educación física para con la inclusión de alumnos con discapacidad, y b) evaluar el efecto diferencial sobre la percepción de autoconfianza en función del sexo, las experiencias y el contacto previo de los profesores y profesoras.

\section{Método}

\section{Participantes}

Ciento y dos $(\mathrm{n}=102)$ profesores y profesoras de último ciclo de educación primaria y primer ciclo de educación secundaria de la Comunitat Valenciana participaron en el presente estudio. En cuanto al sexo, 61 eran hombres (59.8\%) y 42 mujeres (41.2\%), con una edad promedio de $40.47 \pm 9.02$ años (hombres $=41.34 \pm 8.89$, mujeres $=39.17 \pm 9.18$ ). $\mathrm{La}$ experiencia docente de los profesores y profesoras incluidos en el estudio era de $14.87 \pm 9.02$ años (hombres $=15.02 \pm 9.13$, mujeres $=14.63 \pm 8.96$ ).

En lo que se refiere a los datos de experiencias previas de formación en actividades físicas y/o deportes para personas con discapacidad, sólo 38 de los profesores y profesoras participantes habían tenido experiencia formativa previa (37.3\%; hombres $=68.4 \%$, mujeres $=$ $31.6 \%)$. Y en lo que respecta al contacto previo con personas con discapacidad en el ámbito profesional, encontramos experiencias de contacto en 64 de los profesionales participantes (63.4\%; hombres $=56.3 \%$, mujeres $=43.8 \%)$. Todos los profesores firmaron un consentimiento previo a la toma de datos, avalado por el Órgano Evaluador de Proyectos de la Universidad Miguel Hernández de Elche (Referencia DPS.RRV.01.15).

\section{Instrumento}

Se ha procedido a la traducción y estudio de fiabilidad del instrumento denominado SelfEfficacy Scale for Physical Education Teacher Education Majors towards Children with Disabilities (SE-PETE-D), creado y validado por Block et al. (2013) en inglés. Se trata de una 
escala que consta de 4 partes y 3 sub-escalas. Comienza con unas instrucciones acerca de cómo cumplimentar la escala, incluyendo una breve explicación de la teoría de la autoeficacia (Bandura, 1994) y un ejemplo acerca de cómo es la ratio de evaluación de la escala tipo Likert, donde 1 significa "sin confianza” y 5 significa "confío completamente" ante las situaciones planteadas. Las preguntas formuladas se agrupan en situaciones donde el profesor debe atender a la inclusión de un alumno con discapacidad intelectual, física y visual, concretamente acerca de la evaluación de capacidades físicas, enseñanza de habilidades motrices, y organización de juegos deportivos colectivos. La primera parte comienza con una descripción de un estudiante con discapacidad intelectual que los profesores deben incluir en sus clases de EF, debiendo contestar 11 preguntas al respecto. La segunda parte es referida a la inclusión de un alumno con discapacidad física, sobre el que deben contestar a 12 preguntas acerca de cómo se sienten de capaces y confiados para su inclusión en clase; y se finaliza con una tercera situación para con un alumno con discapacidad visual, acerca de quien se deben contestar 10 preguntas. La cuarta y última parte del cuestionario recoge datos demográficos de los participantes tales como edad, sexo o años de experiencia laboral en EF. Para evaluar las experiencias previas de formación en relación al objeto de estudio, se incluyó la pregunta: “¿Has cursado antes algún curso o formación en actividad/educación física adaptada/inclusiva?”, debiendo contestar "si” o "no". Además, para conocer el grado de experiencia previa o contacto con personas con discapacidad, se incluyó la pregunta “¿Has estado involucrado previamente en alguna situación de deporte/educación física adaptada/inclusiva?”, debiendo ser también contestada como "si" o "no".

\section{Procedimiento}

Dada la ausencia de instrumentos válidos y fiables en el contexto español sobre el objeto de estudio, se procedió a la traducción del instrumento SE-PETE-D (Block et al., 2013), al que nos referiremos a partir de ahora como Escala de Autoeficacia en Profesores de Educación Física hacia Alumnos con Discapacidad (EA-PEF-AD). Para asegurar que la escala original se ajustaba a profesores cuya lengua materna es el español, se siguió la técnica de traducción transcultural de Vallerand (1989), realizando para ello pequeñas modificaciones sobre la escala original, especialmente en cuanto a la formulación de las preguntas sobre los datos demográficos. Por ejemplo, se ajustó la pregunta acerca de los años de experiencia en EF, ya que en la escala original se pregunta acerca del año que obtuvo la graduación que habilita para dicha profesión; o cuando se pregunta sobre la participación en formaciones previas sobre educación física inclusiva, preguntándose si incluía también actividad física o educación física adaptada.

La traducción fue efectuada por dos profesionales con experiencia en actividad física y educación física adaptada, la cual fue realizada de forma independiente. Cabe indicar que una traducción literal de la escala original no siempre es efectiva, ya que el significado del contenido debe ajustarse al contexto del país de aplicación. Así, las dos traducciones realizadas fueron comparadas y analizadas por tres profesionales independientes con dominio del inglés y el castellano, y se creó una versión preliminar de la escala, que fue analizada a su vez por tres profesores de educación física en activo. Finalmente, la escala final utilizada para el estudio fue previamente analizada por el equipo investigador y un experto en psicometría. 
La toma de datos fue realizada en 7 de las formaciones realizadas durante el curso académico 2015-16 del Proyecto Incluye-T (Reina et al., 2016), dedicado a la formación de profesores y profesoras en educación física en educación física inclusiva. Así, los profesores participantes, una vez firmado el informe de consentimiento de participación, permanecían todos sentados en un aula y recibían instrucciones del investigador principal acerca de la estructura del cuestionario. En la primera página debían indicar un código anónimo para la codificación de cada participante, a la vez de indicar si era antes (pre) o después de la formación que iban a recibir (post). Resuelta cualquier duda que pudiera haber del cuestionario, los profesores disponían de 15 minutos para su cumplimentación.

\section{Resultados}

Los resultados son presentados en términos de valores medios (M) y desviación estándar (DT). Se procedió a la realización de un análisis de normalidad de Kolmogorov-Smirnov para determinar la distribución normal de los valores de la escala EA-PEF-AD, y sus tres subescalas de discapacidad intelectual, física y visual. La fiabilidad de la escala se testó mediante un análisis de fiabilidad de Cronbach, y un análisis de correlación bivariada de Pearson evaluó la relación entre las diferentes sub-escalas, siendo éstas interpretadas como baja $₫(\mathrm{r} 0.3)$, moderada $(0.3<\mathrm{r} \leq 0.7)$ y alta $(\mathrm{r}>0.7)$ (Salaj, y Markovic, 2011). Con la finalidad de analizar la sensibilidad de la escala para evaluar las diferentes sub-escalas o situaciones de la misma, se ha realizado un análisis de varianza de medidas repetidas, reportando los valores de eta al cuadrado parcial $\left(\eta \mathrm{p}^{2}\right)$ de acuerdo a la siguiente interpretación: por encima de .26, entre 0.26 y 0.02, y menor que 0.02 como alto, medio y bajo respectivamente (Pierce, Block, y Aguinis, 2004).

Para analizar el efecto diferencial de la participación previa en actividades formativas o de contacto en el ámbito de la actividad física y del deporte, se utilizó un análisis de varianza de un factor. Se reportan los valores de estimación de tamaño del efecto siguiendo las recomendaciones de Cohen (1988): $>0.8$, excelente; $<0.8-0.5>$, moderado; $<0.5-0.25>$, bajo; < 0.25, trivial. Todos los datos fueron analizados mediante el Statistical Package for Social Sciences (version 21.0 para Windows, SPSS Inc, Chicago, IL, USA), y el nivel de significación fue establecido en $\mathrm{p}<0.05$.

Contrastada la distribución normal de los valores obtenidos, se confirmó la idoneidad de utilización de estadística paramétrica. El análisis de fiabilidad de Cronbach mostró una fiabilidad total de 0.964 si incluimos en el modelo todos los ítems de la escala, siendo de 0.975 para la sub-escala de discapacidad intelectual, 0.935 para la de discapacidad física, y 0.935 para la de discapacidad visual. El análisis de fiabilidad con los valores de cada sub-escala (discapacidad intelectual, física y visual) muestra una fiabilidada de 0.917. La tabla 1 muestra las correlaciones que se han dado entre las tres dimensiones de la escala.

El análisis de medidas repetidas entre las tres dimensiones de la escala revela la existencia de diferencias significativas $\left[\mathrm{F}(1,101)=33.4 ; \mathrm{p}<0.001 ; \eta \mathrm{p}^{2}=0.25\right.$, moderada). La comparación por pares ad hoc de Bonferroni muestra también diferencias $(\mathrm{p}<0.001)$ entre las sub-escalas de discapacidad intelectual $(3.29 \pm 0.64)$ con respecto a las de discapacidad física (3.03 \pm 0.79$)$ y la de discapacidad visual (2.91 \pm 0.77$)$. 
Tabla 1. Correlaciones entre las tres sub-escalas de la EA-PEF-AD y el valor total de la misma.

\begin{tabular}{lcccc}
\hline & 1. & 2. & 3. & 4. \\
\hline 1. Intelectual & -- & $.647^{* *}$ & $.575^{* *}$ & $.837^{* *}$ \\
2. Física & & -- & $.638^{* *}$ & $.891^{* *}$ \\
3. Visual & & & -- & $.862^{* *}$ \\
4. Total & & & & -- \\
\hline
\end{tabular}

** $\mathrm{p}<0.001$; EA-PEF-AD, Escala de Autoeficacia en Profesores de Educación Física hacia Alumnos con Discapacidad.

El análisis de varianza para los factores formación y contacto previo muestra diferencias significativas en las tres sub-escalas y el valor global de la escala EA-PEF-AD, con tamaños del efecto altos en la formación previa para las sub-escalas de discapacidad intelectual (0.83), física (0.80) y total (0.83), siendo bajo para la de discapacidad visual (0.37). Algo similar ocurre para el contacto previo, pero en esta ocasión con tamaños de efecto moderados para las sub-escalas de discapacidad intelectual (0.50), física (0.53) y total (0.67), y bajo para la de discapacidad visual (0.40). No se ha obtenido diferencia alguna para el factor sexo.

Tabla 2. Análisis de varianza en función de la formación y el contacto previo

\begin{tabular}{lllrrr}
\hline & $\mathrm{Si}(\mathrm{M} \pm \mathrm{SD})$ & No $(\mathrm{M} \pm \mathrm{SD})$ & $\mathrm{F}(\mathrm{gl})$ & Sig. & $D$ \\
\hline Intelectual - Formación & $3.6 \pm 0.6$ & $3.1 \pm 0.6$ & $15.6(100)$ & 0.001 & 0.83 \\
Intelectual - Contacto & $3.4 \pm 0.6$ & $3.1 \pm 0.6$ & $6.3(99)$ & 0.014 & 0.50 \\
Física - Formación & $3.4 \pm 0.7$ & $2.8 \pm 0.8$ & $13.6(100)$ & 0.001 & 0.80 \\
Física - Contacto & $3.2 \pm 0.7$ & $2.8 \pm 0.8$ & $6.6(99)$ & 0.012 & 0.53 \\
Visual - Formación & $3.1 \pm 0.9$ & $2.8 \pm 0.7$ & $5.8(100)$ & 0.017 & 0.37 \\
Visual - Contacto & $3.0 \pm 0.8$ & $2.7 \pm 0.7$ & $5.0(99)$ & 0.028 & 0.40 \\
\hline Total - Formación & $3.4 \pm 0.6$ & $2.9 \pm 0.6$ & $15.2(100)$ & 0.001 & 0.83 \\
Total - Contacto & $3.2 \pm 0.6$ & $2.8 \pm 0.6$ & $8.1(99)$ & 0.005 & 0.67 \\
\hline
\end{tabular}

( $\mathrm{M}=$ media, DT = desviación típica, gl = grados de libertad totales, $d=$ tamaño del efecto de Cohen)

\section{Discusión}

Este trabajo analiza el grado de fiabilidad de la versión española de la Escala de Autoeficacia en Profesores de Educación Física hacia Alumnos con Discapacidad (EA-PEFAD), y la sensibilidad de la misma para poder discriminar entre factores demográficos del profesorado de educación física, tales como el sexo, la formación previa en actividad física y deporte adaptado, o el contacto previo con personas con discapacidad en educación física.

La Escala EA-PEF-AD ha mostrado una alta consistencia interna a tenor de los valores de alpha de Cronbach, mostrándose fiable para evaluar la autoeficacia de profesores de educación física para con la inclusión de alumnos con discapacidad intelectual, física y visual, al igual que han mostrado recientes estudios (Block et al., 2013). Las altas correlaciones obtenidas entre las diferentes sub-escalas muestran que ésta puede ser un instrumento fiable para evaluar el grado de autoeficacia que el profesor tendría a nivel global, si bien el número de profesores incluidos en este estudio debería ser aumentado para poder llevar a cabo un estudio adecuado de validez de la escala en el contexto español. 
La diferenciación entre diferentes situaciones o tipos de discapacidad ha sido un elemento mediador a la hora de evaluar las actitudes hacia la inclusión y la autoeficacia percibida (Block, y Rizzo, 1995; Kowalski, y Rizzo, 1996), habiendo sido sugerido como un factor a controlar o incluir en el diseño de escalas de evaluación (Hutzler et al., 2005), y siendo las de discapacidad física, intelectual y visual las que suponen un mayor reto a la hora de llevar a cabo la inclusión en clases de educación física (Hutzler, 2003). El efecto diferencial que puede suponer atender a cada una de las diferentes situaciones o grupos de discapacidad se ve avalado por las diferencias obtenidas entre las tres dimensiones, especialmente en la comparación por pares entre la discapacidad intelectual con respecto a la física y la visual. No obstante, sigue sin haber un consenso claro en la literatura acerca de qué tipo de discapacidad es percibida como una mayor barrera para su inclusión en EF (Lijuan, Jing, y Lin, 2015), aunque el estudio de la UNESCO denominado United Nations Educational, Scientific and Cultural Organization (Avramidis, y Norwich, 2012) indica que la discapacidad considerada más fácil de incluir es la física, seguida de las dificultades de aprendizaje y del habla, las dificultades conductuales y emocionales severas, la discapacidad visual y auditiva, y por último, la discapacidad intelectual.

Cabe indicar que una de las principales barreras para la inclusión de alumnos con discapacidad en educación física radica precisamente en cómo se sienten de competentes los profesores para poder llevar a cabo ese proceso de una forma satisfactoria (Ammah, y Hodge, 2006; Hardin, 2005; Hodge, 1998; Kowalski, y Rizzo, 1996; LaMaster, Gall, Kinchin, y Siedentop, 1998; Lienert, Sherrill, y Myers, 2001). Existe pues una relación entre la percepción de competencia y las actitudes hacia la inclusión de alumnos con discapacidad en clases de educación física, de manera que aquellos con mayores valores en ambos constructos mostrarían una mayor autoeficacia percibida. Si consideramos la autoeficacia como los sentimientos que las personas tienen sobre su capacidad para desempeñar sus funciones en un entorno que requiere una determinadas demandas (Bandura, 1994), aquellos profesores que perciban una alta autoeficacia de sí mismos no sentirán cuestionada su capacidad para atenderlas y podrían percibir dicha situación como un reto ante el que responder. Por el contrario, los profesores con un bajo nivel de autoeficacia percibida podrían tener incongruencias entre su capacidad y las demandas de la tarea (Hutzler et al., 2005).

Las experiencias previas en cuanto a formación o contacto se presentan pues como un factor mediador en el grado de autoeficacia percibido por parte de los profesores de EF. Así, las actitudes de los docentes hacia la enseñanza de alumnado con diferentes grados de habilidad y discapacidad parece ser un factor clave para una inclusión exitosa (Hodge, y Jansma, 1999, 2000; Folsom-Meek, y Rizzo, 2002), si bien muchos docentes manifiestan cierta incapacidad y falta de apoyos para atender a alumnos con discapacidad en sus clases, a veces porque consideran que no están lo suficientemente preparados para atenderles (Grenier, 2006), cuestión relacionada con el objeto de estudio de la presente investigación. Nos encontramos que aquellos docentes con un alto nivel de competencia percibida y experiencias positivas en la enseñanza con estudiantes con discapacidad suelen mostrar unas mejores actitudes hacia éstos (Folsom-Meek, Nearing, Groteluschen, y Krampf, 1999; Hodge, Davis, Woodard, y Sherrill, 2002; Tripp, y Rizzo, 2006), siendo más partidarios de un modelo de enseñanza inclusivo (DePauw, y Doll-Tepper, 2000; Hodge, Ammah, Casebolt, Lamaster, y O’Sullivan, 2004). 
Una formación específica y conocimientos acerca de la discapacidad son determinantes para la inclusión (Avramidis, Bayliss, y Burden, 2000; Vickerman, y Coates, 2009), de manera que aquellos profesores que no hayan tenido una formación previa adecuada no se verán preparados para atender a la diversidad en el aula (Davis, Kotecki, y Oliver, 2007; Rust, y Sinelnikov, 2010), pudiendo ello estar relacionado con su percepción de autoeficacia a tenor de los resultados obtenidos en la presente investigación.

Las diferencias favorables que se han encontrado en cuanto al grado de autoeficacia percibida en aquellos profesores que han tenido una formación previa, se ve reforzada por aquellos que han tenido también experiencias previas o contacto con la discapacidad, factores que parecen aumentar el grado de confianza del profesor para con la inclusión, y un cambio positivo y favorable hacia la misma (Lijuan et al., 2015). Este tipo de experiencias aumentarían considerablemente las intenciones favorables del profesorado para una educación física inclusiva, sintiéndose más capaces de enfrentarse diariamente al proceso de enseñanza (Oh et al., 2010), al igual que cuando tienen experiencias favorables (Hodge et al., 2004). En este sentido, Hodge, Tannehill y Kluge (2003) confirmaron que los profesores de EF se sentían con más confianza para con la enseñanza de alumnos con discapacidad después de haber asistido a una formación de experiencia práctica; mientras que Tripp, French y Sherrill (1995) demostraron cómo las experiencias previas de trabajo con alumnos con discapacidad estaban altamente relacionadas con las actitudes e intenciones positivas de los profesores de EF hacia la enseñanza de alumnos con discapacidad. Parece pues que los profesores tienen que experimentar sensaciones de sentirse capaces de adaptar su enseñanza para la inclusión, siendo indispensable la experiencia previa con alumnos con discapacidad (De Boer,Pijl, Post, y Minnaert, 2011; Özer et al., 2013).

Como conclusiones, estamos de acuerdo con Ocete-Calvo (2016) que las experiencias docentes vienen influenciadas por la formación específica, de manera que el nivel de conocimientos que el docente posea sobre el tipo de discapacidad a tratar y las adaptaciones necesarias para su inclusión influirá notablemente en las decisiones metodológicas que tome. Los resultados del presente trabajo confirman la idoneidad de llevar a cabo programas formativos para profesores de educación física en activo, fomentando las acciones de contacto o inclusión de participantes con discapacidad. Ello se ve apoyado por los mayores de valores de autoeficacia percibida mostrada por los profesores y profesoras que han participado en programas formativos en relación a la actividad o educación física adaptada y/o inclusiva, así como por parte de aquellos que han tenido experiencias profesionales y de contacto previas.

\section{Agradecimientos}

La toma de datos de este trabajo se ha hecho al amparo del contrato CPE1.15F entre la Universidad Miguel Hernández de Elche y el Comité Paralímpico Español para la formación de profesorado de la Comunidad Valenciana en educación física inclusiva, subvencionado por la Fundación Trinidad Alfonso. 


\section{Referencias}

Ammah, J. O., y Hodge, S. R. (2005). Secondary physical education teachers' beliefs and practices in teaching students with severe disabilities: A descriptive analysis. High School Journal, 89(2), 40-54.

Avramidis, E., y Norwich, B. (2012). Teachers' attitudes towards integration/inclusion: A review of the literature. European Journal of Special Needs Education, 17(2), 129-147.

Avramidis, E., Bayliss, P., y Burden, R. (2000). A survey into mainstream teachers' attitudes towards the inclusion of children with special educational needs in the ordinary school. Teaching and Teacher Education, 16, 277-293.

Bandura, A. (1989). Human agency in social cognitive theory. American Psychologist, 44(9), $1175-1184$.

Bandura, A. (1994). Self-efficacy. En V. S. Ramachaudran (Ed.), Encyclopedia of human behavior (Vol. 4, pp. 71-81). New York: Academic Press.

Block, M. E. (1994). Why all students with disabilities should be included in regular Physical Education. Palaestra, 10(3), 17-24.

Block, M. E., y Obrusnikova, I. (2007). Inclusion in Physical Education: A review of the literature from 1995-2005. Adapted Physical Activity Quarterly, 24, 103-124.

Block, M. E., y Rizzo, T. (1995). Attitudes and attributes of physical educators associated with teaching individuals with severe and profound disabilities. Journal of Association for Persons with Severe Handicaps, 20(1), 80-87.

Block, M.E., Hutzler, Y., Barak, S., y Klavina, A. (2013). Creation and validation of the selfefficacy instrument for physical education teacher education majors toward inclusion. Adapted Physical Activity Quarterly, 29, 184-205.

Davis, R., Kotecki, J. M. H., y Oliver, A. (2007). Responsibilities and training needs of paraeducators in physical education. Adapted Physical Activity Quarterly, 24(1), 70-83.

De Boer, A., Pijl, S., Post, W., y Minnaert, A. (2011). Which variables relate to the attitudes of teachers, parents and peers towards students with special education needs in regular education? Educational Needs, 38(4), 433-448.

DePauw, K.P., y Doll-Tepper, G. (2000). Toward progressive inclusion and acceptance: Myth or reality? The inclusion debate and bandwagon discourse. Adapted Physical Activity Quarterly, 17(2), 135-143.

Folsom-Meek, S. L., Nearing, J. R., Groteluschen, W., y Krampf, H. (1999). Effects of academic major, gender, and hands-on experience on the attitudes of preservice professionals. Adapted Physical Activity Quarterly, 16, 389-402.

Folsom-Meek, S. L., y Rizzo, T. L. (2002). Validating the Physical Educators' attitude toward Teaching Individuals with Disabilities III (PEATID III) survey for future professionals. Adapted Physical Activity Quarterly, 19, 141-154.

Grenier, M. (2006). A social constructionist perspective of teaching and learning in inclusive physical education. Adapted Physical Activity Quarterly, 23, 245-260.

Hardin, B. (2005). Physical education teacher's reflections on preparation for inclusion. Physical Educator, 62(1), 44-56.

Hodge, S. (1998). Prospective physical education teacher's attitudes. Physical Educator, 55(2), 68-77. 
Hodge, S. R., y Jansma, P. (1999). Effects of contact time and location of practicum experiences on attitudes of physical education majors. Adapted Physical Activity Quarterly, 16, 48-63.

Hodge, S., y Jansma, P. (2000). Physical education majors' attitudes toward teaching students with disabilities. Teacher Education and Special Education, 23, 211-224.

Hodge, S. R., Tannehill, D., y Kluge, M. A. (2003). Exploring the meaning of practicum experiences for PETE students. Adapted Physical Activity Quarterly, 20(4), 381-399.

Hodge, S. R., Davis, R., Woodard, R., y Sherrill, C. (2002). Comparison of practicum types in changing preservice teachers' attitudes and perceived competence. Adapted Physical Activity Quarterly, 19(2), 155-171.

Hodge, S.R., Ammah, J.O., Casebolt, K., LaMaster, K., y O’ Sullivan, M. (2004). High school general physical education teachers' behaviors and beliefs associated with inclusion. Sport, Education \& Society, 9, 395-419.

Hutzler, Y., Zach, S., y Gafni, O. (2005). Physical education students' attitudes and selfefficacy towards the participation of children with special needs in regular classes. European Journal of Special Needs Education, 20(3), 309-327.

Jovanovic, L., Kudlacek, M., Block, M.E., y Djordjevic, I. (2014). Self-efficacy of pre-service physical education teacher toward teaching students with disabilities in general physical education classes in Serbia. European Journal of Adapted Physical Activity, 7(2), 32-46.

Kodish, S., Kulinna, P. H., Martin, J., Pangrazi, R., y Darst, P. (2006). Determinants of physical activity in an inclusive setting. Adapted Physical Activity Quarterly, 23, 390409.

Konza, D. (2008). Inclusion of students with disabilities in new times: responding to the challenge. En P. Kell, W. Vialle, D. Konza, y G. Vogl (Eds), Learning and the learner: exploring learning for new times (pp. 38-65). Wollongong: University of Wollongong.

Kowalski, E. M., y Rizzo, T. L. (1996). Factors influencing preservice student attitudes toward individuals with disabilities. Adapted Physical Activity Quarterly, 13, 180-196.

Kozub, F. M., y Lienert, C. (2003). Attitudes toward teaching children with disabilities: Review of literature and research paradigm. Adapted Physical Activity Quarterly, 20(4), 323-346.

LaMaster, K., Kinchin, G., Gall, K., y Siedentop, D. (1998). Inclusion practices of effective elementary specialists. Adapted Physical Activity Quarterly, 15 (1), 64-81.

LOMCE, L. O. 8/2013, de 9 de diciembre, para la mejora de la calidad educativa (2013). Consultada en el BOE, 295.

Lienert, C., Sherrill, C., y Myers, B. (2001). Physical educators' concerns about integrating children with disabilities: A cross-cultural comparison. Adapted Physical Activity Quarterly, 18(1), 1-17.

Lijuan, W., Jing, Q., y Lin, W. (2015). Beliefs of Chinese physical educators on teaching students with disabilities in general physical education classes. Adapted Physical Activity Quarterly, 32(2), 137-155.

Ocete-Calvo, C. (2016). "Deporte inclusivo en la escuela". Diseño y análisis de un programa de intervención para promover la inclusión del alumnado con discapacidad en educación física.Tesis doctoral no publicada, INEF, Universidad Politécnica de Madrid, Madrid, España. 
Oh, H., Rizzo, T., So, H., Chung, D., Park, S., y Lei, Q. (2010). Preservice physical education teachers' attributes related to teaching a student labelled ADHD. Teaching and Teacher Education, 26(4), 885-890.

ONU (2006). United Nations Convention on the Rights of Persons with Disabilities (2006). $\begin{array}{lllllll}\text { Extraído el } & 15 & \text { de junio de } & 2016 & \text { desde }\end{array}$ http://www.un.org/disabilities/convention/conventionfull.

Özer, D., Nalbant, S., Aglami, E., Baran, F., Kaya Samut, P., Aktop, A., y Hutzler, Y. (2013). Physical education teachers' attitudes towards children with intellectual disability: the impact of time in service, gender, and previous acquaintance. Journal of Intellectual Disability Research, 57(11), 1001-1013.

Pierce, C. A., Block, R. A., y Aguinis, H. (2004). Cautionary note on reporting eta-squared values from multifactor ANOVA designs. Educational and Psychological Measurement, 64, 916-924.

Reina, R. (2014). Inclusión en deporte adaptado: dos caras de una misma moneda. Psychology, Society, \& Education, 6(1), 55-67.

Reina, R., Hutzler, Y., Iniguez-Santiago, M. C., y Moreno-Murcia, J. A. (2016). [Attitudes towards inclusion of Spanish physical education students: Relationship with ability beliefs, gender, and previous experiences] Manuscript submitted for publication.

Reina, R., Sierra-Marroquín, B., Garcia-Gómez, B., Fernández-Pacheco, Y., Hemmelmayr, I., García-Vaquero, M. P., Campayo-Piernas, M., y Roldán, A. (2016). "IncluyeT’.Educación física y deporte inclusivo. Elche: Limencop.

Rust, R., y Sinelnikov, O. (2010). Practicum in self-contained environment: Pre-service teacher perceptions of teaching students with disabilities. Physical Educator, 67(1), 33-46.

Salaj, S., y Markovic, G. (2011). Specificity of jumping, sprinting, and quick change-ofdirection motor abilities. Journal of Strength and Conditioning Research,25(5), 12491255.

Taliaferro, A. R., Hammond, L., y Wyant, K. (2015). Preservice physical educators' selfefficacy beliefs toward inclusion: the impact of coursework and practicum. Adapted Physical Activity Quarterly, 32(1), 49-67.

Tripp, A., y Rizzo, T. (2006). Disability labels affect physical educators. Adapted Physical Activity Quarterly, 23(3), 310-326.

Tripp, A., French, R., y Sherrill, C. (1995). Contact theory and attitudes of children in physical education programs toward peers with disabilities. Adapted Physical Activity Quarterly, 12(4), 323-332.

United Nations (2015). Treaty Series: Convention on the Rights of Persons with Disabilities. Dec. 13, 2006, Vol. 2515, p. 3. Extraído el 15 de junio de 2016 desde https://treaties.un.org/doc/Publication/MTDSG/Volume\%20I/Chapter\%20IV/IV15.en.pdf.

Vallerand, R. J. (1989). Vers une méthodologie de validationtrans-culturelle de questionnaires psychologiques: Implications pour la recherche en langue française. Psychologie Canadienne, 30(4), 662-680.

Vickerman, P., y Coates, J. (2009). Trainee and recently qualified physical education teachers' perspectives on including children with special education needs. Physical Education and Sport Pedagogy, 14(2), 137-153. 